\title{
Distribution of $\mathrm{HNCO} \mathbf{5}_{\mathbf{0 5}}-\mathbf{4}_{\mathbf{0 4}}$ in massive star-forming regions
}

\author{
J. Li ${ }^{1,2,3,4}$ J. Z. Wang ${ }^{2,3,4}$, Q. S. Gu ${ }^{2,3}$, and X. W. Zheng ${ }^{2,3}$ \\ 1 Shanghai Astronomical Observatory, Chinese Academy of Sciences, 80 Nandan Road, 200030 Shanghai, PR China \\ e-mail: lijuan@shao.ac.cn \\ 2 Key Laboratory of Radio Astronomy, Chinese Academy of Sciences, 210008 Nanjing, PR China \\ 3 School of Astronomy \& Space Science, Nanjing University, 22 Hankou RD, 210093 Nanjing, PR China \\ ${ }^{4}$ Key Laboratory of Modern Astronomy and Astrophysics (Nanjing University), Ministry of Education, 210093 Nanjing, PR China
}

Received 18 December 2012 / Accepted 6 May 2013

\section{ABSTRACT}

\begin{abstract}
Aims. The goal of this paper is to study the spatial distribution of HNCO in massive star-forming region and to investigate both its spatial association with infrared sources and physical conditions in region of HNCO emission.

Methods. We mapped nine massive star-forming regions in $\mathrm{HNCO} 5_{05}-4_{04}$ with the Purple Mountain Observatory $13.7 \mathrm{~m}$ telescope. The $\mathrm{C}^{18} \mathrm{O}$ maps of these sources were obtained simultaneously.

Results. The HNCO emission shows compact distribution, with the emission peak centered on water masers. Nearly all the HNCO clumps show signs of embedded mid-infrared or far-infrared sources. The FWHM sizes of HNCO clumps are significantly smaller than $\mathrm{C}^{18} \mathrm{O}$ clumps but rather similar to $\mathrm{HC}_{3} \mathrm{~N}$ clumps. We find a good correlation between the integrated intensities, linewidths, and LSR velocities of $\mathrm{HNCO}$ and $\mathrm{HC}_{3} \mathrm{~N}$ emission, implying similar excitation mechanisms for these two species. As such, collisional excitation is likely to be the dominant excitation mechanism for $\mathrm{HNCO} 5_{05}-4_{04}$ emission in galactic massive star-forming regions.
\end{abstract}

Key words. ISM: clouds - ISM: molecules - radio lines: ISM

\section{Introduction}

Interstellar isocyanic acid (HNCO) was first detected in Sgr B2 molecular cloud complex, where it was found to be spatially extended and relatively strong (Snyder \& Buhl 1972; Churchwell et al. 1986; Lindqvist et al. 1995; Kuan \& Snyder 1996; Dahmen et al. 1997). Since its discovery, HNCO has been detected in various molecular clouds, including the dark cloud TMC-1 (e.g. Brown 1981; Jackson et al. 1984), as well as in hot cores in massive star-forming regions (e.g. MacDonald et al. 1996; Helmich \& van Dishoeck 1997). Jackson et al. (1984) proposed that $\mathrm{HNCO}$ was a dense gas tracer due to the coincidence of HNCO emission with regions of high density $\left(n \geq 10^{6} \mathrm{~cm}^{-3}\right)$. Zinchenko et al. (2000) report a detection rate of $70 \%$ in a survey of 81 molecular clouds. HNCO has also been detected in some extragalactic sources (Nguyen-Q-Rieu et al. 1991; Meier \& Turner 2005; Martín et al. 2009).

Within the Galactic center region, obvious different distribution of $\mathrm{HNCO}$ and $\mathrm{C}^{18} \mathrm{O}$, which is thought to be a tracer of the total $\mathrm{H}_{2}$ column density, suggests possible different chemical properties of the different molecular complexes in the center of our Galaxy (Dehmen et al. 1997; Lindqvist et al. 1995; Martín et al. 2008). Based on the morphology of the emission and the $\mathrm{HNCO}$ abundance with respect to $\mathrm{H}_{2}$, several authors hypothesized that $\mathrm{HNCO}$ could be a good tracer of interstellar shocks (e.g., Zinchenko et al. 2000; Meier \& Turner 2005; Minh \& Irvine 2006). Martín et al. $(2008,2009)$ conducted a multitransition study of 13 molecular clouds towards the Galactic center and concluded that the $\mathrm{HNCO} / \mathrm{CS}$ abundance ratio might provide a useful tool for distinguishing between the influence of shocks and radiation activity in the nuclear regions of galaxies. Rodríguez-Fernández et al. (2010) test the hypothesis by observing a low-mass molecular outflow where the chemistry is dominated by shocks. Their results indicate that shocks can actually produce the $\mathrm{HNCO}$ abundance measured in galactic nuclei, providing a solid basis to previous suggestions that the extended HNCO in galactic nuclei could trace large-scale shocks.

Chemical models have also been developed to investigate how HNCO forms. Both gas-phase reaction (e.g., Turner et al. 1999) and formation routes on grain surfaces (e.g. Hasegawa \& Herbst 1993; Garrod et al. 2008) have been used to model HNCO abundance. Tideswell et al. (2010) find that HNCO is inefficiently formed when only gas-phase formation pathways are considered in the chemical network, and surface routes are needed to account for its abundance. Quan et al. (2010) have reproduced the abundances of $\mathrm{HNCO}$ and its isomers in cold and warm sources using gas-grain simulation, which contains both gas-phase and grain-surface syntheses.

Zinchenko et al. (2000) mapped three molecular clouds in $\mathrm{HNCO}$ transitions and found that $\mathrm{HNCO}$ emission is compact and centrally peaked. Because they are limited by angular resolutions, the size of HNCO clouds, the relationships with infrared sources, as well as the dominant excitation mechanism of HNCO emission remain unknown. From this consideration it is clear that high-sensitivity observations are needed to better understand the physical condition and chemical properties of HNCO clouds.

In this paper we present large-scale mapping observations of $\mathrm{HNCO}$ and $\mathrm{C}^{18} \mathrm{O}$ toward strong sources detected in Zinchenko et al. (2000) with the Purple Mountain Observatory $13.7 \mathrm{~m}$ (PMODLH $13.7 \mathrm{~m}$ ) telescope. We first introduce the observations and data reduction in Sect. 2. In Sect. 3, we present the observational results. In Sect. 4, we discuss implication of our observations on the excitation mechanism and chemistry of HNCO, followed by a summary in Sect. 5 . 
Table 1. Source list.

\begin{tabular}{lcccc}
\hline \hline Source name & RA(J2000) & $\operatorname{Dec}(\mathrm{J} 2000)$ & $D(\mathrm{kpc})$ & Ref. \\
\hline G121.30+0.66 & $00: 36: 47.51$ & $63: 29: 02.1$ & 1.2 & 1 \\
Orion KL & $05: 35: 14.47$ & $-05: 22: 27.56$ & 0.414 & 2 \\
W44 & $18: 53: 18.50$ & $01: 14: 56.7$ & 3.7 & 3 \\
W51M & $19: 23: 43.86$ & $14: 30: 29.4$ & 5.41 & 4 \\
ON1 & $20: 10: 09.14$ & $31: 31: 37.4$ & 2.57 & 5 \\
DR21S & $20: 39: 00.80$ & $42: 19: 29.8$ & 1.5 & 6 \\
W75OH & $20: 39: 01.01$ & $42: 22: 49.9$ & 1.5 & 6 \\
S158 & $23: 13: 44.84$ & $61: 26: 50.71$ & 2.65 & 7 \\
S140 & $22: 19: 19.04$ & $63: 18: 50.4$ & 0.76 & 8 \\
\hline
\end{tabular}

References. (1) Plume et al. (1992); (2) Menten et al. (2007); (3) Solomon et al. (1987); (4) Sato et al. (2010); (5) Rygl et al. (2010); (6) Rygl et al. (2011); (7) Moscadelli et al. (2009); (8) Hirota et al. (2008).

\section{Observations and data reductions}

We performed mapping observations of HNCO $5_{05}-4_{04}$ (109.905 GHz) and $\mathrm{C}^{18} \mathrm{O} 1-0(109.782 \mathrm{GHz})$ lines simultaneously with the PMODLH 13.7 m located in Delingha, China in January 2011. The main beam size is about 55", and the pointing accuracy is estimated to be better than $9^{\prime \prime}$. A new cryogenically cooled 9 -beam SIS receiver $(3 \times 3$ with a separation of $174^{\prime \prime}$ between the centers of adjacent beams) working in the $85-115 \mathrm{GHz}$ band were employed. A fast-Fourier transform spectrometer (FFTS) of 16384 channels with a bandwidth of $1 \mathrm{GHz}$ was used for each beam, supplying a velocity resolution of about $0.21 \mathrm{~km} \mathrm{~s}^{-1}$. Typical system temperatures were around 150-300 K, depending on the weather conditions. Observations were made in on-the-fly mode with nine beams. The telescope drifted in azimuth (with a rate of $20^{\prime \prime} \mathrm{s}^{-1}$ ) and stepped in elevation (with a scan step of 15"). Several maps were made and later combined to lower the rms noise levels. The mapping size was $10^{\prime} \times 10^{\prime}$ for most sources. Mapping centers of all our sources are listed in Table 1.

Most of the sources were selected from dense cores showing strong HNCO $5_{05}-4_{04}$ emission in the surveys of Zinchenko et al. (2000). W44, S140, and DR21S are also included in our sample. W44 is a molecular cloud interacting with a supernovae remnant (SNR), which provides a promising environment for production of HNCO. Both S140 and DR21S are massive starforming regions with strong $\mathrm{HC}_{3} \mathrm{~N}$ emission ( $\mathrm{Li}$ et al. 2012). Table 1 lists information on the sources that have been mapped in this study. The distances were determined from an extensive literature search. Trigonometric parallax distances were used, if available, or otherwise photometric distances or kinematic distances based on rotation curve of Fich, Blitz \& Stark (1989) were used.

The data processing was conducted using Gildas package ${ }^{1}$. A least-square fit to baselines in the spectra was carried out with the first-order polynomial. The baseline slopes were removed for all the sources. The individual spectra were averaged, and the resulting spectra were Hanning-smoothed to improve the signalto-noise ratio $(\mathrm{S} / \mathrm{N})$ of the data. The line parameters were obtained by Gaussian fitting. We express the results in the unit of main beam brightness temperature $\left(T_{\mathrm{mb}}\right)$ assuming the main beam efficiencies of 0.5 (Chen et al. 2010, 2012).

To search for the mid-infrared (MIR) emission of young stellar objects (YSOs), we used the Midcourse Space Experiment

1 http://wWw.iram.fr/IRAMFR/GILDAS
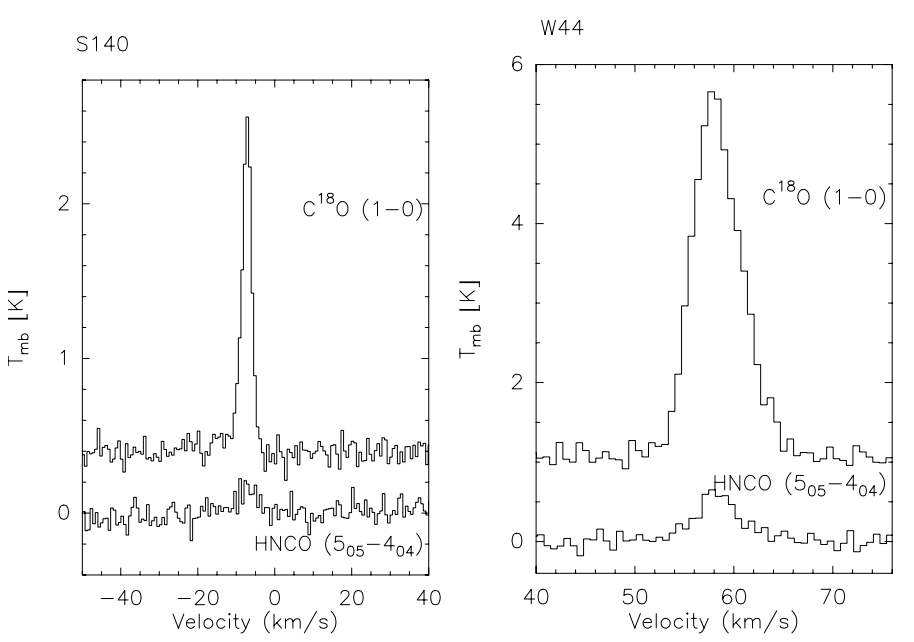

Fig. 1. HNCO $5_{05}-4_{04}$ and $\mathrm{C}^{18} \mathrm{O} 1-0$ spectra of S140 and W44 at $(0,0)$. Identification of the transitions is given to the right of each lines.

(MSX) Galactic plane survey between 6 and $25 \mu \mathrm{m}$ at $18^{\prime \prime}$ spatial resolution (Price et al. 2001). For S140, where no MSX data are available, the AKARI/IRC source catalog (Murakami et al. 2007; Onaka et al. 2007) of the AKARI all-sky survey is used to search for MIR emission ( 9 and $18 \mu \mathrm{m}$ ) with a spatial resolution of about 6". To search for the far-infrared (FIR) emission of YSOs, we used the AKARI/FIS bright source catalog (Kawada et al. 2007) centered at 65,90,140 and $160 \mu \mathrm{m}$, with spatial resolutions ranging from $37^{\prime \prime}$ to $61^{\prime \prime}$.

\section{Observing results}

Figure 1 presents the HNCO $5_{05}-4_{04}$ and $\mathrm{C}^{18} \mathrm{O} 1-0$ spectra of S140 and W44. Both of them are newly detected in HNCO. The derived line parameters of HNCO $5_{05}-4_{04}$ and $\mathrm{C}^{18} \mathrm{O} 1-0$ at $(0,0)$ are presented in Tables 2 and 3 , respectively, including main beam brightness temperature $\left(T_{\mathrm{mb}}\right)$, integrated line intensity $\left(\int T_{\mathrm{mb}} \mathrm{d} v\right)$, LSR velocity, and full-width half-maximum (FWHM) linewidth of HNCO $5_{05}-4_{04}$ emission. All the parameters are determined from Gaussian fitting. Our derived parameters are similar but not identical to results of Zinchenko et al. (2000) because their main beam size is only two-thirds of our observations.

HNCO $5_{05}-4_{04}$ maps were obtained for nine sources. In Fig. 2, we present contour maps of HNCO $5_{05}-4_{04}$ and $\mathrm{C}^{18} \mathrm{O}$ overlayed on the MSX $21.3 \mu \mathrm{m}$ image on a linear scale. Except for Orion KL, eight other sources were mapped with the same telescope and toward the same positions with $\mathrm{HNCO}$ in the $\mathrm{HC}_{3} \mathrm{~N} 10-9$ transition $\left(90.979 \mathrm{GHz}\right.$ ) (Li et al. 2012). The $\mathrm{HC}_{3} \mathrm{~N}$ contour maps were also overlayed for comparison. The MIR and FIR sources from AKARI catalogs are marked, along with the positions of water masers. Compared with the widespread $\mathrm{C}^{18} \mathrm{O}$ distribution, $\mathrm{HNCO}$ emission shows a rather compact distribution, with emissions concentrating on water masers. The HNCO emission peaks are offset from the $\mathrm{C}^{18} \mathrm{O}$ in Orion A, W51M, and $\mathrm{S} 158$. The recently improved resolution of infrared observations allow us to better understand the relationship between $\mathrm{HNCO}$ clumps and infrared sources. Nearly all the HNCO clumps are spatially coincident with MIR or FIR sources, which usually trace the warm dust emission of the cocoons of the OB star central exciting source (Crowther \& Conti 2003). The morphology and spatial distribution of $\mathrm{HNCO} 5_{05}-4_{04}$ emission are similar to the $\mathrm{HC}_{3} \mathrm{~N} 10-9$ transition, which is a dense gas tracer (e.g. Li et al. 2012). These results imply that HNCO emission is emitted 
Table 2. Observational results of $\mathrm{HNCO} 5_{05}-4_{04}$ transition.

\begin{tabular}{lcccccc}
\hline \hline Source name & $\begin{array}{c}T_{\mathrm{mb}} \\
(\mathrm{K})\end{array}$ & $\begin{array}{c}\int T_{\mathrm{mb} v} \mathrm{~d} v \\
\left(\mathrm{~K} \mathrm{~km} \mathrm{~s}^{-1}\right)\end{array}$ & $\begin{array}{c}V_{\mathrm{LSR}} \\
\left(\mathrm{km} \mathrm{s}^{-1}\right)\end{array}$ & $\begin{array}{c}F W H M \\
\left(\mathrm{~km} \mathrm{~s}^{-1}\right)\end{array}$ & $\begin{array}{c}R_{\mathrm{HNCO}} \\
(\mathrm{pc})\end{array}$ & $\begin{array}{c}\theta_{\mathrm{HNCO}} \\
(\mathrm{arcsec})\end{array}$ \\
\hline G121.30+0.66 & $0.24(.04)$ & $0.62(.08)$ & $-17.09(.13)$ & $2.36(.40)$ & 0.17 & 28 \\
Orion KL & $0.96(.12)$ & $7.10(.26)$ & $7.94(.11)$ & $5.99(.34)$ & 0.03 & 11 \\
W44 & $0.58(.08)$ & $3.24(.22)$ & $58.27(.16)$ & $5.20(.46)$ & 0.67 & 37 \\
W51M & $0.48(.06)$ & $4.80(.22)$ & $55.75(.22)$ & $9.52(.51)$ & 1.44 & 55 \\
W51M-west & $0.46(.06)$ & $5.46(.22)$ & $64.58(.23)$ & $11.28(.48)$ & - & - \\
ON1 & $0.22(.06)$ & $1.12(.12)$ & $11.79(.26)$ & $4.63(.59)$ & 0.45 & 36 \\
DR21S & $0.12(.03)$ & $0.46(.06)$ & $-2.17(.21)$ & $3.50(.56)$ & 0.29 & 39 \\
W75OH-north & $0.26(.04)$ & $1.04(.10)$ & $-3.25(.17)$ & $3.75(.46)$ & - & - \\
W75OH & $0.38(.04)$ & $1.60(.10)$ & $-3.31(.13)$ & $4.02(.32)$ & 0.50 & 68 \\
S158 & $0.44(.06)$ & $1.96(.12)$ & $-56.15(.13)$ & $4.23(.29)$ & 0.69 & 53 \\
S140 & $0.16(.06)$ & $1.08(.16)$ & $-7.10(.47)$ & $6.03(1.21)$ & 0.15 & 41 \\
\hline
\end{tabular}

Table 3. Observational results of $\mathrm{C}^{18} \mathrm{O} 1-0$ transition.

\begin{tabular}{lcccccc}
\hline \hline Source name & $\begin{array}{c}T_{\mathrm{mb}} \\
(\mathrm{K})\end{array}$ & $\begin{array}{c}\int T_{\mathrm{mb}} \mathrm{d} v \\
\left(\mathrm{~K} \mathrm{~km} \mathrm{~s}^{-1}\right)\end{array}$ & $\begin{array}{c}V_{\mathrm{LSR}} \\
\left(\mathrm{km} \mathrm{s}^{-1}\right)\end{array}$ & $\begin{array}{c}F W H M \\
\left(\mathrm{~km} \mathrm{~s}^{-1}\right)\end{array}$ & $\begin{array}{c}R_{\mathrm{HNCO}} \\
(\mathrm{pc})\end{array}$ & $\begin{array}{c}\theta_{\mathrm{HNCO}} \\
(\mathrm{arcsec})\end{array}$ \\
\hline G121.30+0.66 & $1.52(.06)$ & $3.32(.06)$ & $-17.33(.02)$ & $2.04(.05)$ & 0.43 & 73 \\
Orion KL & $1.76(.08)$ & $7.72(.14)$ & $8.84(.04)$ & $4.13(.10)$ & 0.16 & 78 \\
W44 & $4.42(.08)$ & $26.66(.26)$ & $58.19(.03)$ & $5.68(.07)$ & 1.40 & 78 \\
W51M & $2.70(.08)$ & $31.94(.06)$ & $56.49(.06)$ & $11.10(.11)$ & 2.20 & 84 \\
W51M-west & $1.21(.08)$ & $25.34(0.40)$ & $60.90(.16)$ & $19.66(.34)$ & - & - \\
ON1 & $1.78(.06)$ & $6.70(.12)$ & $11.35(.03)$ & $3.53(.07)$ & 0.74 & 59 \\
DR21S & $2.92(.06)$ & $9.60(.10)$ & $-2.37(.01)$ & $3.09(.04)$ & 0.52 & 71 \\
W75OH-north & $3.22(.04)$ & $9.66(.08)$ & $-3.69(.01)$ & $2.82(.03)$ & - & - \\
W75OH & $2.06(.04)$ & $11.22(.12)$ & $-2.95(.02)$ & $3.45(.04)$ & 0.63 & 87 \\
S158 & $1.38(.10)$ & $7.98(.20)$ & $-56.18(.07)$ & $5.43(.17)$ & 1.27 & 98 \\
S140 & $2.08(.06)$ & $6.54(.14)$ & $-7.23(.03)$ & $2.95(.07)$ & 0.40 & 108 \\
\hline
\end{tabular}

from a small volume of warmer and denser gas located nearer to the embedded objects than the $\mathrm{C}^{18} \mathrm{O}$ emission.

The size of clouds is characterized by using the beam deconvolved angular diameter and linear radius of a circle with the same area as the half peak intensity:

$\theta_{\text {transition }}=2\left(\frac{A_{1 / 2}}{\pi}-\frac{\theta_{\text {beam }}^{2}}{4}\right)^{1 / 2}$,

$R_{\text {transition }}=D\left(\frac{A_{1 / 2}}{\pi}-\frac{\theta_{\text {beam }}^{2}}{4}\right)^{1 / 2}$,

where $A_{1 / 2}$ is the area within the contour of half peak intensity, $\theta_{\text {beam }}$ the FWHM beam size, and $D$ the distance of the source. The deconvolved linear FWHM sizes and angular diameter of $\mathrm{HNCO}$ and $\mathrm{C}^{18} \mathrm{O}$ clouds are presented in Tables 2 and 3, respectively. The deconvolved linear sizes of HNCO clouds range from 0.05 to $2.88 \mathrm{pc}$, where the lowest value comes from Orion KL, and the highest comes from W51M. The HNCO clouds are significantly smaller than those of $\mathrm{C}^{18} \mathrm{O}$ for nearly all the sources except $\mathrm{W} 75 \mathrm{OH}$, in which the size of $\mathrm{HNCO}$ cloud is comparable to that of $\mathrm{C}^{18} \mathrm{O}$.

Below we give comments on individual sources.

G121.30+0.66. We detected HNCO $5_{05}-4_{04}$ emission with $T_{\mathrm{mb}}$ of $0.24 \mathrm{~K}$. The HNCO clump has a centrally condensed structure, and the emission peak coincides with the MIR emission.

Orion KL. Orion KL is the closest massive star-forming region (414 pc; Menten et al. 2007). Several molecular components, such as the hot core, compact and extended ridges, and several luminous IR sources or radio sources are associated with Orion KL. The nature of the sources responsible for the luminous IR emission is still poorly known and much debated. Other than being powered by an embedded central heating source (e.g., Kaufman et al. 1998), an interesting alternative explanation for this region's energetics is a protostellar merger event that released a few times $10^{47}$ erg of energy about 500 years ago (Bally \& Zinnecker 2005; Zapata et al. 2011; Bally et al. 2011). Our observations show that the HNCO peak was displaced from the $\mathrm{C}^{18} \mathrm{O}$ peak and is centered on MSX $21.3 \mu \mathrm{m}$ emission peak. The $\mathrm{HNCO} / \mathrm{C}^{18} \mathrm{O}$ intensity ratio at $(0,0)$ is close to unity, while there are fewer than 0.25 in other sources. As shock enhancement of HNCO has been detected in L1157 molecular outflow (Rodríguez-Fernández et al. 2010), the relatively high $\mathrm{HNCO} / \mathrm{C}^{18} \mathrm{O}$ intensity ratio seems to favor the idea that the chemistry in Orion KL should be shock-driven with much higher temperature than the chemistry in a typical hot core (Favre et al. 2011), and should produce high abundances of HNCO (Zinchenko et al. 2000).

W44. W44 is an SNR with shell-like morphologies (Dubner et al. 2000; Jones et al. 1993). It is adjacent to a giant molecular cloud that it is suspected of interacting with (Wootten 1981; Denoyer 1983). It contains OH $1720 \mathrm{MHz}$ masers that are attributed to dense, shocked gas (Lockett et al. 1999). The MSX image of W44 consists of an SNR and dust emission heated by embedded YSOs. If it is correct that HNCO is enhanced in the presence of shocks due to its injection into the gas phase from the grain mantles (Zincheko et al. 2000), HNCO would be expected in molecular clouds interacting with SNRs. Strong HNCO emission was detected with $T_{\mathrm{mb}}$ of $0.58 \mathrm{~K}$. The $\mathrm{HNCO}$ emission concentrates mainly around the YSO. More sources should be observed to investigate whether HNCO is abundant in molecular clouds interacting with SNRs.

W51M. This is a strong HNCO $5_{05}-4_{04}$ emission source with $T_{\mathrm{mb}}$ of $0.48 \mathrm{~K}$. Two cores were seen in HNCO clouds. 

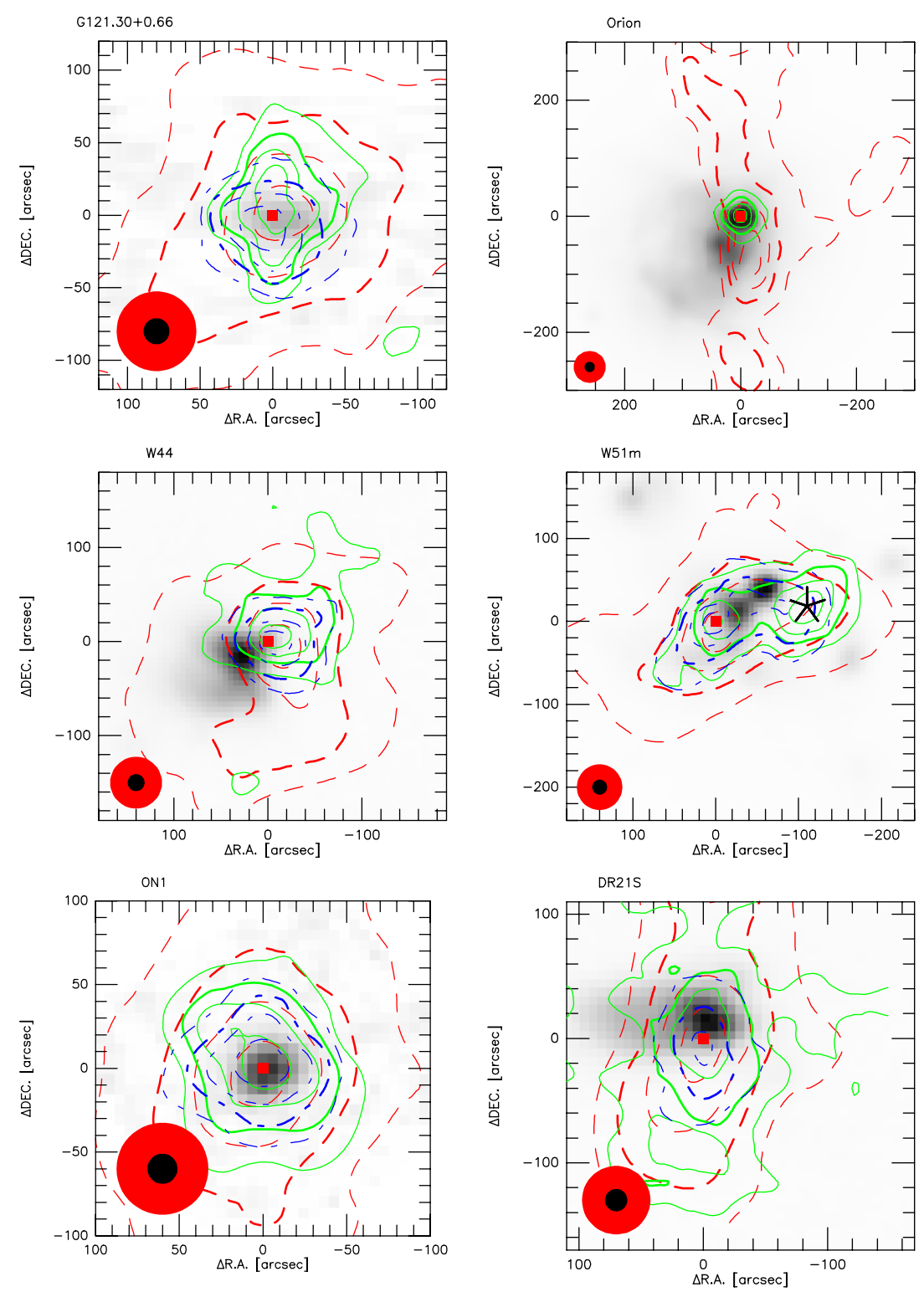

Fig. 2. Contour maps of $\mathrm{HNCO}$ (green solid line), $\mathrm{HC}_{3} \mathrm{~N}$ (blue dotted line), and $\mathrm{C}^{18} \mathrm{O}$ (red dashed line) superimposed on the MSX $21.3 \mu \mathrm{m}$ image for observing sources. The contour levels are 30\%, 50\%, 70\%, and 90\% of the map peak, reported in Table 3 (see Col. 3 for HNCO, Col. 7 for $\mathrm{C}^{18} \mathrm{O}$, see Col. 3 in Table 3 of $\mathrm{Li}$ et al. (2012) for $\mathrm{HC}_{3} \mathrm{~N}$ ). The heavy lines represent $50 \%$ of the map peak. " $\star$ " is used to mark the position of the AKARI FIR source. " $*$ " is used to mark the position of the AKARI MIR source in S140. Red filled squares are used to mark the position of water masers. The FWHM beam size for molecular lines (the big, red circle) and mid-infrared (the small, black circle) observations are shown in the lower left of the maps. (A color version of this figure is available in the online journal.)

The western peak, W51m-west, is stronger than the eastern peak in $\mathrm{HNCO}$ emission, but weaker than the eastern peak in $\mathrm{HC}_{3} \mathrm{~N}$ and $\mathrm{C}^{18} \mathrm{O}$ emission. W51m-west is centered on the AKARI FIR sources, while the eastern peak is coincident with water masers and near to MIR sources.

ON1. We detected HNCO $5_{05}-4_{04}$ emission with $T_{\mathrm{mb}}$ of 0.22 K. Similar to G121.30+0.66, the HNCO emission morphology has a centrally condensed structure, with the emission peak coinciding with the MIR emission.

DR21S. DR21S is a strong $\mathrm{HC}_{3} \mathrm{~N}$ emission source in Li et al. (2012). This is a massive clump in the Cygnus X region. HNCO was detected in DR21S with $T_{\mathrm{mb}}$ of $0.12 \mathrm{~K}$. The $\mathrm{HNCO}$ emission peak is near to the MIR emission peak position.

$\mathrm{W} 75 \mathrm{OH}$. W75OH is also a massive clump in the Cygnus $\mathrm{X}$ region, which hosts three massive dense cores at an early stage in their evolution. We detected HNCO $5_{05}-4_{04}$ emission with
$T_{\mathrm{mb}}$ of $0.38 \mathrm{~K}$. Two HNCO cores were seen in $\mathrm{W} 75 \mathrm{OH}$. The southern peak is brighter than the northern peak in HNCO emission. The southern peak is near the AKARI FIR source, while the northern clump, W75OH-north, is near the strong MIR emission. Csengeri et al. (2011) report detecting velocity shears and low-velocity shocks in $\mathrm{W} 75 \mathrm{OH}$. Since HNCO is thought to be enhanced by low-velocity shocks, high-resolution observations of this source are expected to test this hypothesis by comparing the spatial distribution of $\mathrm{HNCO}$ and $\mathrm{N}_{2} \mathrm{H}^{+}$convergent flows.

S158. S158 is also referred to as NGC 7538 (Moreno \& Chavarría-K 1986). We detected HNCO $5_{05}-4_{04}$ emission with $T_{\mathrm{mb}}$ of $0.44 \mathrm{~K}$. Two clumps were seen in $\mathrm{HNCO}$ and $\mathrm{HC}_{3} \mathrm{~N}$ clouds, with the stronger one coincident with the AKARI MIR source.

S140. S140 shows strong $\mathrm{HC}_{3} \mathrm{~N}$ emission in Li et al. (2012). HNCO $5_{05}-4_{04}$ was marginally detected with $T_{\mathrm{mb}}$ of $0.16 \mathrm{~K}$. 

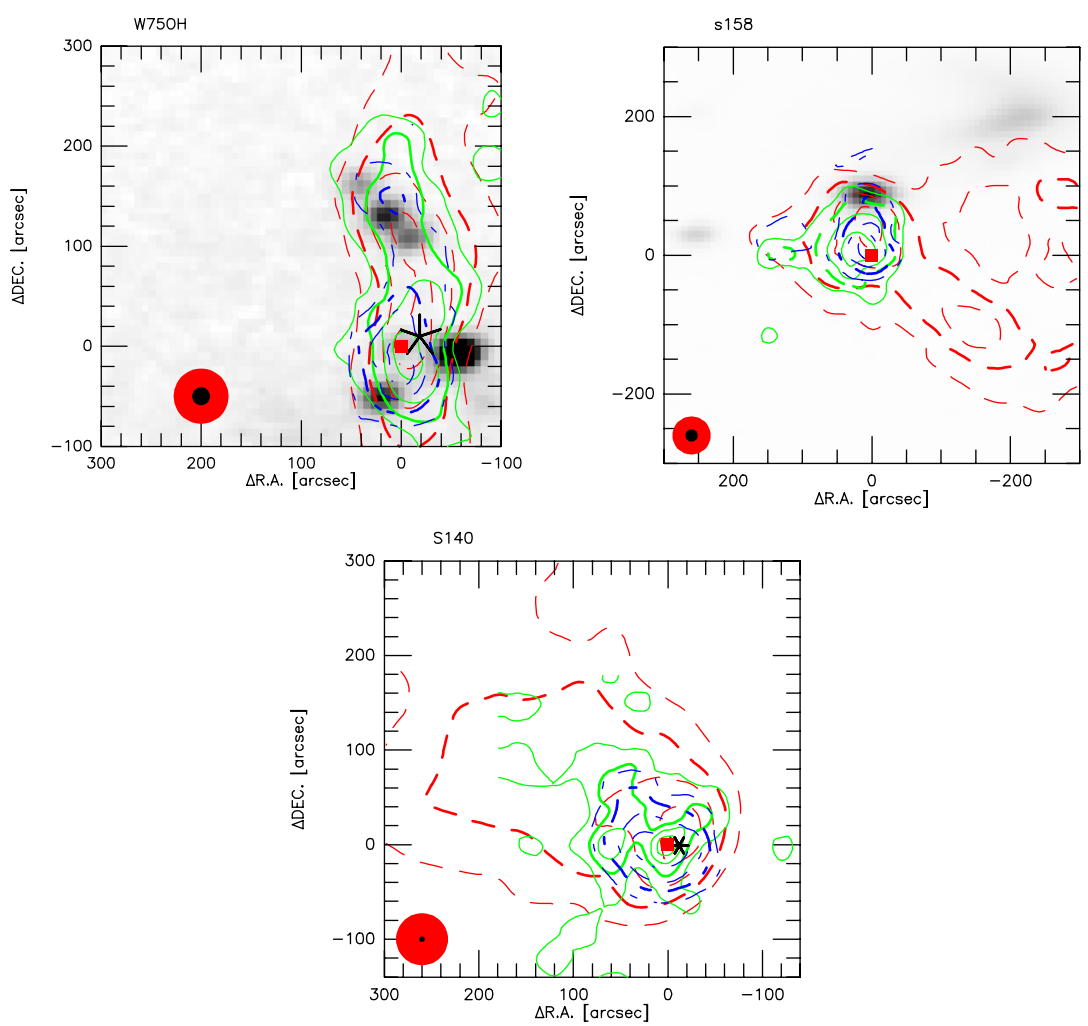

Fig. 2. continued.

Two HNCO cores were seen with low S/N. One of them is associated with the AKARI MIR sources, while another one is starless.

\section{Discussions}

Churchwell et al. (1985) mapped 14 transitions of HNCO toward Sgr B2. Analysis of population distribution indicates the FIR radiation from warm dust is likely to be responsible for the excitation of HNCO. They proposed that the most likely excitation mechanism of $\mathrm{HNCO}$ in $\mathrm{Sgr} \mathrm{B} 2$ was radiative rather than collisional. In this case HNCO was a good probe of the FIR radiation field but not of gas properties such as density and kinetic temperature. The physical environment of hot cores differ from the Galactic center, in which the gas density $\left(10^{3}-10^{4} \mathrm{~cm}^{-3}\right)$ is much lower than in hot cores, thus the dominant excitation mechanism might be different. Zinchenko et al. (2000) explain the $K_{-1}>0$ ladders of Orion KL with radiative excitation. For the $K_{-1}=0$ transitions with a larger source size, they propose that the radiative excitation would become inefficient, and the collisional excitation may dominate.

In Fig. 3, we present plots that compare integrated intensities, linewidths, FWHM size, and LSR velocities of HNCO $5_{05}-4_{04}$ and $\mathrm{HC}_{3} \mathrm{~N} 10-9$ (Li et al. 2012) ${ }^{2}$. The source names are also labeled in the figure. Good correlation is found for integrated intensities $\left(r_{\text {corr }}=0.63\right)$ and FWHM sizes $\left(r_{\text {corr }}=0.98\right)$ of $\mathrm{HNCO}$ and $\mathrm{HC}_{3} \mathrm{~N}$ clumps. The linewidths of HNCO clumps also agree well with $\mathrm{HC}_{3} \mathrm{~N}$ clumps $\left(r_{\text {corr }}=0.92\right)$, with the only exception being $\mathrm{S} 140$, which is possibly caused by the marginal detection of HNCO $(<3 \sigma)$ in this source (see Fig. 1). The velocity difference between $\mathrm{HNCO}$ and $\mathrm{HC}_{3} \mathrm{~N}$ are within $3 \sigma$ for all sources except W51M, which is possibly related to

\footnotetext{
2 We obtain $T_{\mathrm{mb}}$ of $\mathrm{HC}_{3} \mathrm{~N}$ by assuming the main beam efficiencies of 0.5 .
}

the complex structure of this source. The obvious correlations between line parameters, as well as the similar morphology of $\mathrm{HNCO}$ and $\mathrm{HC}_{3} \mathrm{~N}$ clumps, suggest that these two molecule lines trace a similar volume of gas. The critical density of $\mathrm{HC}_{3} \mathrm{~N} 10-9$ is $10^{6} \mathrm{~cm}^{-3}$ (e.g., Chung et al. 1991), which is comparable to the critical density of $\mathrm{HNCO} 5_{05}-4_{04}$. The dominant excitation mechanism for $\mathrm{HC}_{3} \mathrm{~N}$ is collisional excitation, thus collisional excitation is likely to be the dominant excitation mechanism for $\mathrm{HNCO} K_{-1}=0$ emission in galactic massive star-forming regions.

In sources mapped by Zinchenko et al. (2000), HNCO emission peaks are significantly displaced from any known IR sources. However, observations at that time were severely limited by the low resolution of infrared observations. With the greatly improved spatial resolution and sensitivity of IR observations, we found that nearly all the HNCO clumps in our observations are associated with MIR or FIR emission. Thus HNCO should be produced in hot gas. This is consistent with Bisschop et al. (2007), in which HNCO was classified as "hot" molecules based on rotation diagram analysis. Sanhueza et al. (2012) observed a sample of infrared dark clouds (IRDCs) and find that HNCO profiles show no evidence of being a tracer of shocks in most of the sources, but only in two sources, which represent $10 \%$ of the sources with HNCO detection, the HNCO spectrum presents a blue wing that is also observed in $\mathrm{SiO}$. For results present here, we found possible shock enhancement of HNCO in two sources (Orion KL and W75OH). We conclude that HNCO is produced in a warm environment, while shock could enhance the HNCO abundance.

\section{Summary and prospects}

In this paper we present $\mathrm{HNCO} 5_{05}-4_{04}$ mapping observations of nine massive star-forming regions with the PMODLH $13.7 \mathrm{~m}$ telescope. The $\mathrm{C}^{18} \mathrm{O} 1-0$ maps of these sources are obtained 

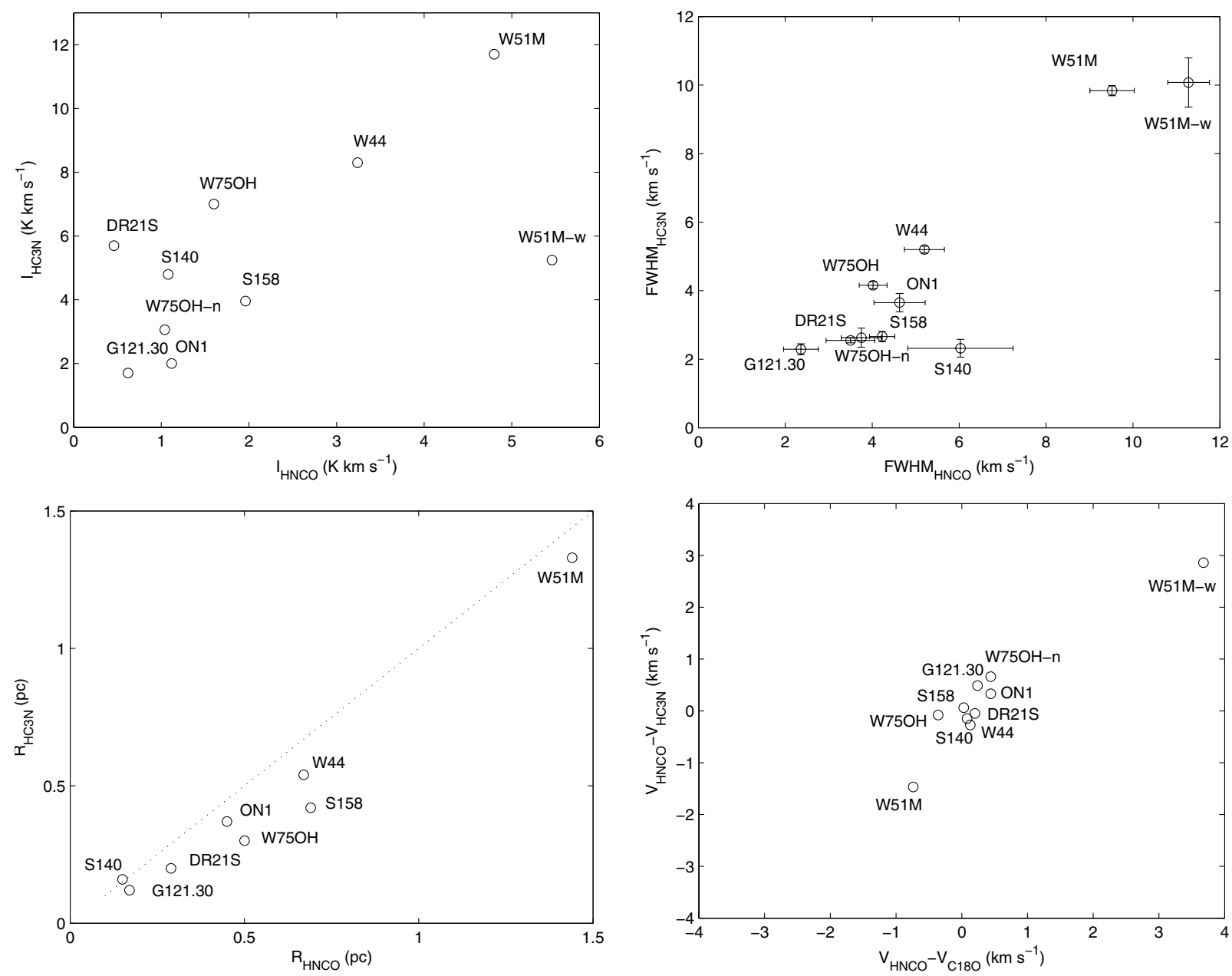

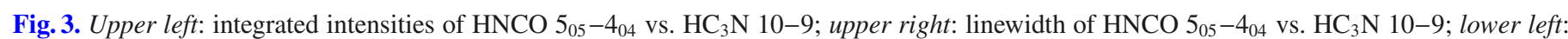
size of $\mathrm{HNCO} 5_{05}-4_{04} \mathrm{vs}$. $\mathrm{HC}_{3} \mathrm{~N} 10-9$. The dotted line has a slop of 1 ; lower right: LSR velocity difference between $\mathrm{HNCO}_{55}-4_{04}$ and $\mathrm{HC}_{3} \mathrm{~N}$ $10-9$ vs. LSR velocity difference between $\mathrm{HNCO} 5_{05}-4_{04}$ and $\mathrm{C}^{18} \mathrm{O} 1-0$. The source names were also labeled in the figure.

simultaneously. We used MSX and AKARI satellite data to search for infrared emission from YSOs and investigated the spatial relationship between HNCO clumps and infrared sources.

We found good correlations between line parameters of $\mathrm{HNCO}$ and $\mathrm{HC}_{3} \mathrm{~N}$ emission, which imply similar excitation mechanisms for these two molecules. The HNCO clumps are much smaller than the $\mathrm{C}^{18} \mathrm{O}$ clumps and comparable to the $\mathrm{HC}_{3} \mathrm{~N}$ clumps. Thus collisional excitation is likely to be the dominant excitation mechanism for $\mathrm{HNCO} 5_{05}-4_{04}$ transition in galactic massive star-forming regions.

We found that HNCO emission is compact and centrally condensed. Nearly all the HNCO clumps show signs of embedded infrared emission, supporting the idea that HNCO is a "hot" molecule. Future high-resolution observations of $\mathrm{W} 75 \mathrm{OH}$ are expected to test the hypothesis that HNCO is enhanced by lowvelocity shock by comparing the spatial distribution of HNCO and $\mathrm{N}_{2} \mathrm{H}^{+}$convergent flows.

PMO is carrying out a $\mathrm{CO},{ }^{13} \mathrm{CO}$, and $\mathrm{C}^{18} \mathrm{O} 1-0$ survey toward the Galactic plane with the DLH 13.7 m telescope. HNCO $5_{05}-4_{04}$ could be observed simultaneously within the $1 \mathrm{GHz}$ band, which would enable us to obtain a spatial distribution of strong HNCO emission in the Galactic plane and to better study the chemical properties of HNCO.

Acknowledgements. We thank the refree for the helpful comments and constructive suggestions. This work is partly supported by the China Ministry of Science and Technology under the State Key Development Program for Basic Research (2012CB821800), and partly supported by the Natural Science Foundation of China under grants of 11103006,10833006 and 10878010 . We would like to thank the Key Laboratory of Radio Astronomy, Chinese Academy of Sciences. We are very grateful to the staff of Qinghai Station of Purple Mountain Observatory for their assistance with the observations and data reductions. This research made use of data products from the Midcourse Space Experiment. This research is based on observations with AKARI, a JAXA project with the participation of ESA. This research made use of the NASA/IPAC Infrared Science Archive, which is operated by the Jet Propulsion Laboratory, California Institute of Technology, under contract with the National Aeronautics and Space Administration.

\section{References}

Bally, J., \& Zinnecker, H. 2005, AJ, 129, 2281

Bally, J. Cunningham, N. J., Moeckel, N., et al. 2011, ApJ, 727, 113

Bisschop, S. E., Jorgensen, J. K., van Dishoeck, E. F., \& de Wachter, E. B. M. 2007, A\&A, 465, 913

Brown, R. L. 1981, ApJ, 248, 119

Chen, X., Shen, Z.-Q., Li, J. J., et al. 2010, ApJ, 710, 150

Chen, X., Ellingsen, S. P., He, J.-H., et al. 2012, ApJS, 200, 5

Chung, H. S., Kameya, O., \& Morimoto, M. 1991, JKAS, 24, 217

Churchwell, E., Wood, D., Myers, P. C., \& Myers, R. V. 1986, ApJ, 305, 405

Crowther, P. A., \& Conti, P. S. 2003, MNRAS, 343, 143

Csengeri, T., Bontemps, S., Schneider, N., et al. 2011, ApJ, 740, 5

Dahmen, G., Huettemeister, S., Wilson, T. L., et al. 1997, A\&AS, 126, 197

Denoyer, L. K. 1983, ApJ, 264, 141

Dubner, G. M., Velaázquez, P. F., Goss, W. M., \& Holdaway, M. A. 2000, AJ, 120,1933 
J. Li et al.: Distribution of HNCO $5_{05}-4_{04}$ in massive star-forming regions

Favre, C., Despois, D., Brouillet, N., et al. 2011, A\&A, 532, A32

Fich, M., Blitz, L., \& Stark, A. A. 1989, ApJ, 342, 272

Garrod, R. T., Weaver, S. L. W., \& Herbst, E. 2008, ApJ, 682, 283

Hasegawa, T. I., \& Herbst, E. 1993, MNRAS, 261, 83

Helmich, F. P., \& van Dishoeck, E. F. 1997, A\&AS, 124, 205

Jackson, J. M., Armstrong, J. T., \& Barett, A. H. 1984, ApJ, 280, 608

Jones, L. R., Smith, A., \& Angellini, L. 1993, MNRAS, 265, 631

Kaufman, M. J., Hollenbach, D. J., \& Tielens, A. G. G. M. 1998, ApJ, 497, 276

Kawada, M., Baba, H., Barthel, P. D., et al. 2007, PASJ, 59, 389

Kuan, Y. J., \& Snyder, L. E. 1996, ApJ, 470, 981

Li, J., Wang, J. Z., Gu, Q. S., Zhang, Z.-Y., \& Zheng, X. W. 2012, ApJ, 745, 47

Lindqvist, M., Sandqvist, A., Winnberg, A., Johansson, L. E. B., \& Nyman, L.-A. 1995, A\&AS, 113, 257

Lockett, P., Gauthier, E., \& Elitzur, M. 1999, ApJ, 511, 235

MacDolnald, G. H., Gibb, A. G., Habing, R. J., \& Millar, T. J. 1996, A\&AS, 119,333

Martin, S., Requena-Torres, M. A., Martin-Pintado, J., \& Mauersberger, R. 2008, ApJ, 678, 245

Martin, S., Martin-Pintado, J., \& Mauersberger, R. 2009, ApJ, 694, 610

Meier, D. S., \& Turner, J. L. 2005, ApJ, 618, 259
Menten, K. M., Reid, M. J., Forbrich, J., \& Brunthaler, A. 2007, A\&A, 474, 515

Minh, Y. C., \& Irvine, W. M. 2006, New A, 11, 59

Moreno, M. A., \& Chavarría-K, C. 1986, A\&A, 161, 130

Murakami, H., Baba, H., Barthel, P., et al. 2007, PASJ, 59, 369

Nguyen-Q-Rieu, Henkel, C., Jackson, J. M., \& Mauersberger, R. 1991, A\&A, 241,33

Onaka, T., Matsuhara, H., Wada, T., et al. 2007, PASJ, 59, 401

Price, S. D., Egan, M. P., Carey, S. J., et al. 2001, AJ, 121, 2819

Quan, D. H., Herbst, E., Osamura, Y., \& Roueff, E. 2010, ApJ, 725, 2101

Rodríguez-Fernández, N. J., Tafalla, M., Gueth, F., \& Bachiller, R. 2010, A\&A, 516, A98

Sanhueza, P., Jackson, J. M., Foster, J. B., et al. 2012, ApJ, 756, 60

Snyder, L. E., \& Buhl, D. 1972, ApJ, 177, 619

Tideswell, D. M., Fuller, G. A., Millar, T. J., \& Markwick, A. J. 2010, A\&A, 510, A 85

Turner, B. E., Terzieva, R., \& Herbst, E. 1999, ApJ, 518, 699

Wootten, A. 1981, ApJ, 245, 105

Zapata, L. A., Schmid-Burgk, J., \& Menten, K. M. 2011, A\&A, 529, A24

Zinchenko, I., Henkel, C., \& Mao, R. Q. 2000, A\&A, 361, 1079 